\title{
Exérese de cálculo salivar em glândula submandibular: relato de caso
}

\author{
Salivary calculus excision in the submandibular gland: case report \\ Exéreses de cálculo salivar em glândulas submandibulares: reporte de caso
}

Recebido: 23/08/2021 | Revisado: 29/08/2021 | Aceito: 02/09/2021 | Publicado: 03/09/2021

\author{
Gustavo Paiva Custódio \\ ORCID: https://orcid.org/0000-0001-6893-369X \\ Centro Universitário de Volta Redonda, Brasil \\ E-mail: gustavopaivacustodio@gmail.com \\ Carlos Henrique Silveira de Castro \\ ORCID: https://orcid.org/0000-0003-1583-0595 \\ Universidade Federal da Bahia, Brasil \\ E-mail: carloshsilveirac@ hotmail.com \\ Cesar Feitoza Bassi Costa \\ ORCID: https://orcid.org/0000-0002-5040-6602 \\ Centro Universitário de Volta Redonda, Brasil \\ E-mail: cesar_bassi@hotmail.com \\ Ana Carolina Silva Mendes \\ ORCID: https://orcid.org/0000-0002-9366-5102 \\ Centro Universitário de Volta Redonda, Brasil \\ E-mail: anamendes.odontologia@gmail.com \\ Gabriel Oliveira Santos \\ ORCID: https://orcid.org/0000-0002-9314-0410 \\ Centro Universitário de Volta Redonda, Brasil \\ E-mail: gabrielsantosunifoa@gmail.com
}

\begin{abstract}
Resumo
Cálculos salivares ou sialolitos são corpos calcificados que se desenvolvem no interior do canal salivar, por meio do acúmulo de sais de cálcio ao redor da luz do ducto, acometendo a glândula submandibular, embora também ocorram nas glândulas sublinguais e parótidas. Essas desordens são manifestadas em pequenos tamanhos, podendo, em alguns casos, atingir grandes proporções. Anatomicamente, o tortuoso e ascendente canal da glândula submandibular (Ducto de Wharton) e a qualidade de sua secreção mucoide espessa são fatores intrínsecos para o surgimento de cálculo salivar. Esses cálculos podem aparecer em qualquer faixa etária, sendo mais comuns em adultos jovens e de meiaidade. O objetivo deste trabalho é discutir um caso clínico de cálculo salivar exposto, sintomático, acometendo o ducto de Wharton do lado direito, em uma paciente de 56 anos de idade, tratado através de remoção cirúrgica simples. A sialolitíase pode aparecer de forma assintomática, porém também pode expor episódios de diminuição do fluxo salivar, dor e edema da glândula afetada com episódios de infecção. A gravidade pode variar a depender do grau de obstrução e da pressão negativa produzida dentro da glândula. $\mathrm{O}$ tratamento poderá ser conservador ou cirúrgico, levando em consideração a glândula afetada e o tamanho do cálculo. Concluiu-se que a conduta mais eficaz no manejo da lesão é através de remoção cirúrgica por acesso intraoral e estas desordens são diagnosticadas primordialmente através do exame clínico, sendo de extrema importância o conhecimento acerca das patologias que envolvem a cavidade oral.
\end{abstract}

Palavras-chave: Cálculo salivar; Sialolitíase; Glândula salivar.

\begin{abstract}
Salivary calculi or sialoliths are calcified bodies that develop inside the salivary canal, through the accumulation of calcium salts around the ductal lumen, affecting the submandibular gland, although they also occur in the sublingual and parotid glands. These disorders are manifested in small sizes and can, in some cases, reach large proportions. Anatomically, the tortuous and ascending canal of the submandibular gland (Wharton's duct) and the quality of its thick mucoid secretion are intrinsic factors for the appearance of salivary calculus. These calculations can appear in any age group, being more common in young and middle-aged adults. The aim of this paper is to discuss the clinical case of exposed, symptomatic salivary calculus affecting the right side of Wharton's duct in a 56-year-old patient treated with simple surgical removal. Sialolithiasis can appear asymptomatic, but it can also show episodes of decreased salivary flow, pain and swelling of the affected gland with episodes of infection. The severity can vary depending on the degree of obstruction and the negative pressure produced inside the gland. Treatment may be conservative or surgical, taking into account the affected gland and the size of the stone. It was concluded that the most effective conduct in the management of the lesion is through surgical removal through intraoral access and these disorders are primary through clinical examination, and knowledge about the pathologies that involve the oral cavity is extremely important.
\end{abstract}

Keywords: Salivary calculation; Sialolithiasis; Salivary gland. 


\begin{abstract}
Resumen
Los cálculos salivales o sialolitos son cuerpos calcificados que se desarrollan en el interior del canal salival, por acumulación de sales de calcio alrededor de la luz ductal, afectando a la glándula submandibular, aunque también ocurren en las glándulas sublingual y parótida. Estos trastornos se manifiestan en tamaños pequeños y pueden, en algunos casos, alcanzar grandes proporciones. Anatómicamente, el canal tortuoso y ascendente de la glándula submandibular (conducto de Wharton) y la calidad de su espesa secreción mucoide son factores intrínsecos para la aparición de cálculos salivales. Estos cálculos pueden aparecer en cualquier grupo de edad, siendo más común en adultos jóvenes y de mediana edad. El objetivo de este trabajo es discutir un caso clínico de cálculo salival sintomático expuesto que afecta el lado derecho del conducto de Wharton en un paciente de 56 años tratado con extirpación quirúrgica simple. La sialolitiasis puede parecer asintomática, pero también puede mostrar episodios de disminución del flujo salival, dolor e hinchazón de la glándula afectada con episodios de infección. La gravedad puede variar según el grado de obstrucción y la presión negativa producida dentro de la glándula. El tratamiento puede ser conservador o quirúrgico, teniendo en cuenta la glándula afectada y el tamaño del cálculo. Se concluyó que la conducta más efectiva en el manejo de la lesión es la extirpación quirúrgica por acceso intraoral y estos trastornos se diagnostican principalmente a través de la exploración clínica, siendo de suma importancia el conocimiento de las patologías que involucran la cavidad bucal.
\end{abstract}

Palabras clave: Cálculo de saliva; Sialolitiasis; Glándula saliva.

\title{
1. Introdução
}

Cálculos salivares são corpos calcificados que se desenvolvem no interior do canal salivar (Neville et al., 2009; Torres et al., 2019). Sua etiologia ainda não é bem esclarecida, mas algumas teorias explicam que seja pela deposição de sais de cálcio em um aglomerado de restos de epitélio do canal descamado, bactérias, muco e corpos estranhos ao redor da luz do ducto (Landgraf et al., 2005; Neville et al., 2009; Pereira et al., 2020).

Esta condição não possui relação com doenças de ordem sistêmica (Neville et al., 2009). Alguns estudos relacionam com fatores locais, como traumas, processos de desordens salivares como tumor de Kuttner que por meio do processo inflamatório, pode comprometer o fluxo salivar, obstruindo o ducto e alterar a qualidade da saliva (Lindenblatt et al., 2007; Silveira et al., 2005; Starling et al., 2012).

Segundo Neville (2009), anatomicamente, de forma sinuosa e ascendente, o ducto de Wharton e a qualidade de sua secreção mucoide mista ser viscosa e espessa, são fatores intrínsecos para o surgimento do cálculo salivar neste canal. A glândula submandibular é a mais acometida, embora também ocorram nas glândulas sublinguais, parótidas e em menor proporção, nas glândulas salivares menores (Silveira et al., 2005; Starling et al., 2012; Souza et al., 2021).

Essas desordens são manifestadas em pequenos tamanhos, podendo, em alguns casos, atingir grandes proporções. Variam de $1 \mathrm{~mm}$ a $10 \mathrm{~mm}$, normalmente. Alguns podem até passar de $10 \mathrm{~mm}$, podendo passar e atingir até $30 \mathrm{~mm}$, sendo considerados sialolitos gigantes (Castro et al., 2021; Jaeger et al., 2013).

São comumente diagnosticadas por meio de exames físicos e radiografias oclusais, apresentando uma área radiopaca, com sua radiopacidade interferida pela composição do cálculo (Castro et al., 2021; Souza et al., 2021).

Entretanto, as características clínicas dessa patologia, são bem marcantes e envolvem cálculos maciços, em formato ovoide, fusiforme ou cilíndrico de coloração amarelada, dependendo da constituição do cálculo. Caso esse cálculo esteja na porção terminal do ducto, pode estar exposto na cavidade bucal e por meio de exame físico, pode ser palpado abaixo da mucosa, uma vez que haverá um aumento de volume nesta região e ser até mesmo, visualizado, eliminando assim, a necessidade de exposição do paciente ao exame radiográfico (Landgraf et al., 2005; Neville et al, 2009; Rodrigues et al., 2017).

O tratamento pode ocorrer por eliminação espontânea ou por bioestimulação, também terapêuticas conservadoras por meio do uso de anti-inflamatórios e antibióticos. Observa-se com frequência taxas de sucesso quando o caso é abordado por intervenção cirúrgica, para tal ato, deve ser levado em consideração a glândula afetada, a localização e o tamanho do cálculo para a escolha da melhor técnica cirúrgica (Landgraf et al., 2005; Jaeger et al., 2013; Silva et al., 2020; Silveira et al., 2005). 
O objetivo deste trabalho é discutir um caso clínico de cálculo salivar exposto, afetando a porção terminal do ducto de Wharton do lado direito de uma paciente do sexo feminino, de 56 anos, abordando suas características clínicas e o seu tratamento cirúrgico simples.

\section{Metodologia}

Refere-se a um relato de caso, caracterizado como um estudo exploratório, descritivo e qualitativo (Pereira et al., 2018).

Foram seguidos os princípios éticos de pesquisa envolvendo seres humanos, sendo preservada a identidade do voluntário e fornecido todos os esclarecimentos sobre o tratamento e após concordância do paciente a assinatura do Termo de Consentimento Livre Esclarecido (TCLE), consentindo o tratamento, a divulgação das imagens e dados coletados durante o atendimento clínico e os que constavam no prontuário, sendo que o mesmo poderia desistir da participação na pesquisa a qualquer instante.

\section{Relato de Caso}

Paciente do sexo feminino, 56 anos, compareceu ao ambulatório de cirurgia e traumatologia bucomaxilofacial, com queixa de dor em assoalho de boca principalmente em momentos de alimentação, edema, sem sangramento e relatava ainda, vermelhidão no local.

Ao exame clínico foi observado aumento de volume em assoalho de boca, fenestração em ducto salivar de Wharton do lado direito, exposição de cálculo salivar, à palpação havia grande mobilidade da estrutura local (Figura 1).

Figura 1: Aspecto inicial da lesão.

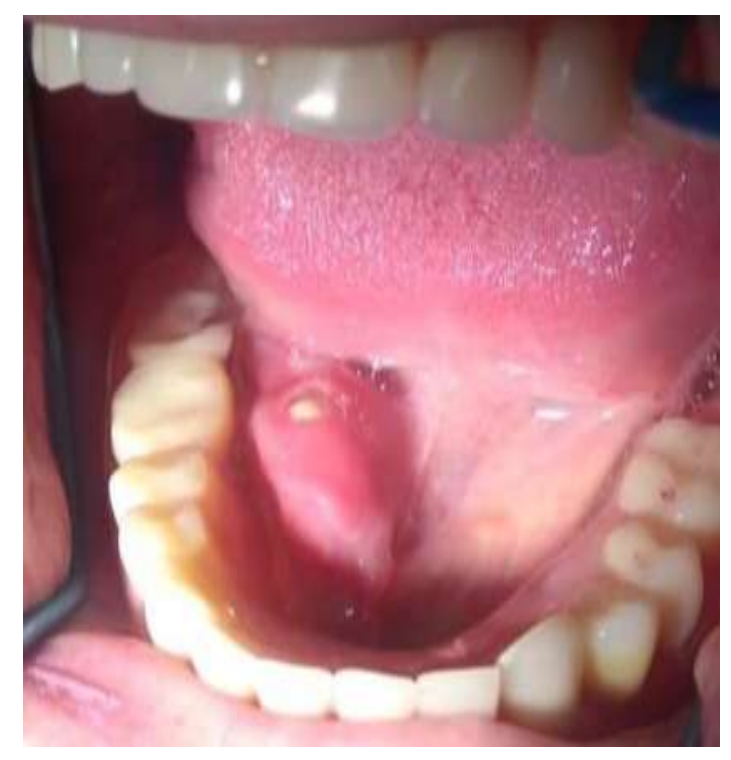

Fonte: Autores.

Foi realizado procedimento cirúrgico para exérese deste cálculo salivar extenso, com infiltração de lidocaína $2 \%$ com epinefrina 1:200.000 em assoalho de boca, incisão superficial com lâmina 15 e divulsão da mucosa do assoalho bucal utilizando pinça mosquito curva, expondo o cálculo salivar em toda sua extensão, evitando incisar abaixo do cálculo , buscando evitando-se lesionar artéria lingual ou nervo lingual, os quais cursam em continuidade ao ducto de Warthon, conseguindo-se assim, sua completa remoção (Figura 2). 
Figura 2: Cálculo submandibular removido.

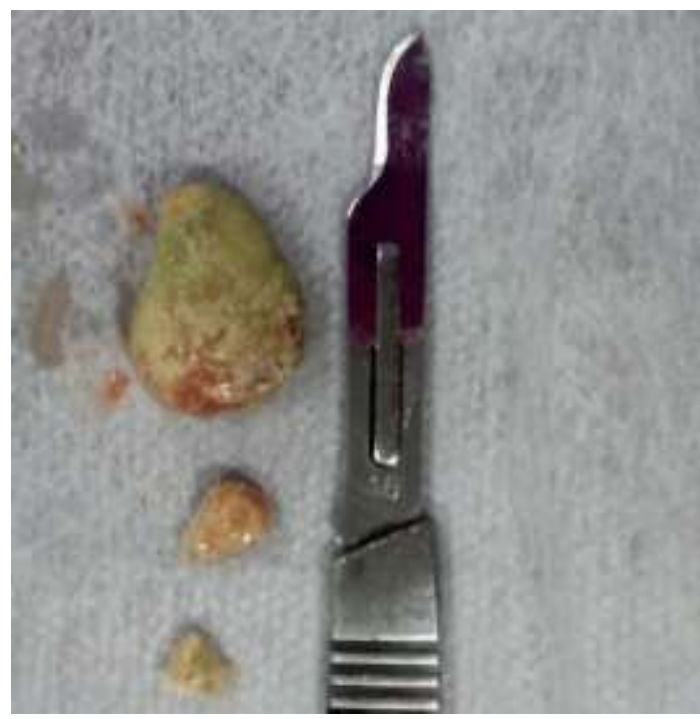

Fonte: Autores.

Foi realizado em seguida, a instalação de dreno para reconstrução do ducto salivar, para assim, permitir a epitealização e formação de um novo orifício de saída de conteúdo salivar do ducto.

Figura 3: Dreno em região de assoalho bucal, após remoção do cálculo salivar.

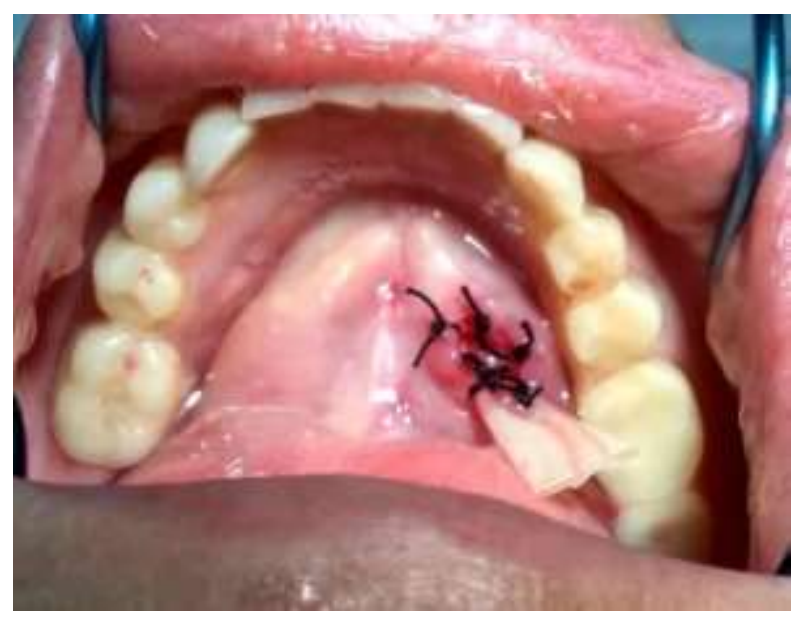

Fonte: Autores.

O dreno foi removido com 07 dias, com a cicatrização dentro dos padrões de normalidade e sem nenhuma queixa do paciente. Paciente foi indicado quanto aos cuidados e necessidade de proservação durante um longo período. 
Figura 4: Acompanhamento de 2 anos.

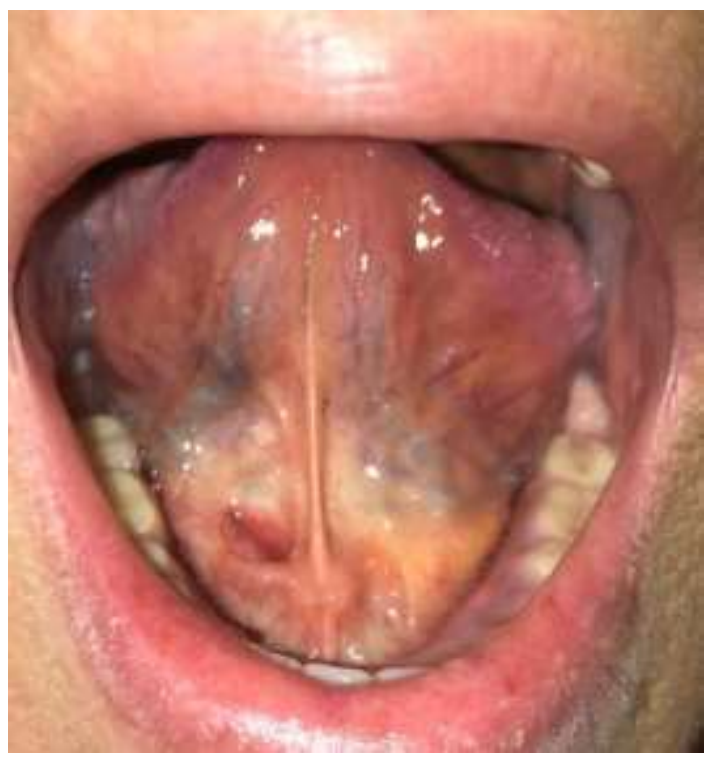

Fonte: Autores.

Atualmente, o caso encontra-se com acompanhamento de 2 anos e possui completa funcionalidade da glândula salivar, não apresenta qualquer alteração de sensibilidade local, sem nenhum quadro de obstrução ductal ou outra complicação local.

\section{Discussão}

Segundo Bodner (2002) a sialolitíase é uma doença comum das glândulas salivares, que é definida pela presença de cálculos nas glândulas ou nos ductos salivares. Os cálculos, também conhecidos como sialolitos, são formados por glicoproteínas, mucopolissacarídeos, restos celulares, carbonato-apatia, sais de cálcio, e diferentes tipos de fosfato, magnésio, ferro e zinco (Cobos, et al., 2009; Pereira et al., 2020; Rodrigues et al., 2017).

Segundo Starling (2012), esses cálculos podem aparecer em qualquer faixa etária, sendo mais comuns em adultos jovens e de meia-idade, com predileção pelo sexo masculino sem predileção por raça. Contrariando-se da literatura, o caso relatado foi em uma paciente do sexo feminino.

O aumento da alcalinidade da saliva, infecção ou inflamação ou até mesmo um trauma físico no ducto ou glândula são fatores predisponentes à formação do cálculo (Folchinim \& Stolz, 2016). A maioria dos cálculos tem origem na glândula submandibular, seu acometimento fica em torno de $80-95 \%$ dos casos, isso é explicado devido a anatomia do ducto de Wharton, por ser longo e tortuoso, concomitante com a ação da gravidade, que dificulta o fluxo salivar e facilita a sua obstrução (Guijarro, et al., 2012; Hong, et al.,2003; Souza et al., 2021), corroborando com o caso apresentado neste estudo.

Um exame clínico cuidadoso e as histórias médica e odontológica bem realizadas são fundamentais para o diagnóstico da sialolitíase. O aumento de volume é o sinal mais frequente do paciente com essa alteração. Usualmente, o paciente relata dor e inchaço da glândula durante os períodos de refeição ou quando há estímulos (Rodrigues et al., 2017; Siddiqui, 2002), essas características assemelham-se com as encontradas neste relato de caso.

O exame radiográfico bidimensional serve para auxiliar o diagnóstico. O que pode ser explicado, pelo grau de mineralização dos cálculos (Lustmann et al.,1990; Starling, et al., 2012). Além disso, pode não ser necessário a realização do exame de imagem, visto que ele é um exame complementar, não sendo justificado a submissão do paciente a expor-se a radiação, sendo que, as características no caso deste relato de caso são totalmente claras do diagnóstico de cálculo salivar. 
Em alguns casos extremos é necessário utilizar tecnologias mais avançadas, como tomografia computadorizada, sialografia, ultrassonografia, cintilografia e ressonância magnética, para ter uma maior precisão de localização e mensuração de tamanho (Mandel \& Alfi, 2012; Souza et al., 2021), neste caso não houve a necessidade devido a exposição já citada do cálculo salivar.

De acordo com estudos feitos por Neto (2012) o tratamento é definido a partir da localização e do tamanho do cálculo salivar, podendo ser conservador ou cirúrgico. Para aqueles próximos ao óstio, o cateterismo e a dilatação de conduto facilitam e permitem a sua remoção; os localizados na metade anterior do ducto necessitam intervenção cirúrgica; e os que estão na porção posterior do ducto ou intraglandulares obrigam geralmente a remoção total da glândula envolvida. $\mathrm{O}$ caso relatado, soma-se a literatura devido a abordagem cirúrgica que foi realizada.

Cálculos menores podem ser "ordenhados" bimanualmente através da saída do ducto (Folchini \& Stolz, 2014; Souza et al., 2021). Porém, caso a calcificação for grande, é indicado a exérese do cálculo ou até mesmo da glândula salivar (Alkurt \& Peter, 2009; Rodrigues et al., 2017).

Contudo, abordagens cirúrgicas envolvendo a glândula salivar podem trazer complicações, como uma fístula salivar, de difícil resolução e prognóstico desfavorável, comprometimento na inervação local, esteneose do ducto, fibrose na região do ducto (Folchini \& Stolz, 2014; Souza et al., 2021). Neste caso, não houve complicações pós-cirúrgica.

É importante ressaltar que a remoção dos cálculos salivares é fundamental para recompor a microbiota oral, visto que obstruções do ducto por longos períodos podem causar danos ao ácino glandular, resultando numa permanente diminuição ou mesmo ausência de secreção salivar. Esta diminuição ou ausência de secreção pode predispor a glândula a recorrentes infecções, o que algumas vezes, requer uma sialadenectomia (Hardy, 1966; Lutsmann et al., 1990; Pereira et al., 2020; Torres et al., 2019).

O prognóstico é favorável, embora os sialolitos podem apresentar altas taxas recidiva ou, em caso de persistência da obstrução, causar lesão do componente parenquimatoso da glândula e causar sialoadenite, no entanto, na maioria dos casos, as glândulas afetadas funcionam normalmente após a remoção da lesão (Rodrigues et al., 2017; Torres et al., 2019). Para este estudo, a paciente encontra-se em acompanhamento de 2 anos, sem sinais de recidiva.

\section{Considerações Finais}

Concluiu-se que é válido ressaltar a importância do conhecimento quanto as alterações patológicas que acometem a cavidade oral, para que seja realizado o diagnóstico correto e consequente o tratamento adequado. O sinal clínico bastante evidente do sialólito na glândula submandibular é a tumefação e desconforto no assoalho bucal, sendo o diagnóstico estabelecido principalmente através do exame clínico, podendo em alguns casos, lançar mão de exames complementares.

A escolha do tratamento pelo profissional está diretamente ligada à localização do cálculo salivar e sua dimensão, onde pode-se optar por uma terapia não invasiva ou intervenção cirúrgica. A terapia cirúrgica não demonstra danos maiores ao paciente, sendo a mais indicada.

Ademais, para os próximos trabalhos, sugere-se que sejam abordadas novas formas de tratamento, com ênfase principalmente em condutas não cirúrgicas.

\section{Referências}

Bodner, L. (2002). Giant salivary gland calculi: Diagnostic imaging and surgical management. Oral Surg Oral Med Oral Pathol, St. Louis, 94(2), 320-323.

Castro, C. C. L. P., Rodrigues, E. D. R., Vasconcelos, B. C. E., \& Moreira, T. C. A. (2021). Abordagem intrabucal para exérese de sialolito na glândula sublingual: relato de caso. Arch Health Invest, 10(3), 427-430. 
Cobos, M. R., Muñoz, Z. C., \& Diaz A. (2009). Sialolitosenconductos y glándulassalivales: Revisión de literatura. Avances en Odontoestomatologia, 25(6), 311-317.

Folchini, S., Stolz A. B. (2016). Sialolitos na glândula submandibular: relato de caso. Odontol Clín9Cient, 15(1), 67-71.

Hong, K.H., Yang Y.S. (2003). Sialolithiasis in the sublingual gland. J Laryngol Otol, 117(11),905-7.

Jaeger, F., Andrade, R., Alvarenga, R. L., Galizes, B. F., \& Amaral, M. B. F. (2013). Sialolito gigante no ducto da glândula submandibular. Revista Portuguesa de Estomatologia, Medicina Dentária e Cirurgia Maxilofacial. 54(1), 33-36.

Landgraf, H., Assis, A. F., Klüppel, L. E., Oliveira, C. F., \& Gabrielli, M. A. C. (2005). Extenso sialolito no ducto da glândula submandibular: relato de caso. Revista de Cirurgia e Traumatologia Buco-maxilo-facial Camaragibe, 6(2), 29-34.

Lindeblatt, R. C., Santos, J. B., Alves, D. R., Lourenço, S. Q. C., \& Dias, E. P. (2007). Sialoadenite esclerosante crônica (tumor de kuttner): relato de caso clínico. J Bras Patol Med Lab, 43(5), 381-384.

Lustmann, J., Regev E., \& Melamed Y. (1990). Sialolithiasis. A survey on 245 patients and a review of the literature. Int J Oral Maxillofac Surg, 19(3), 13538 .

Mandel, L., \& Alfi D. (2012). Diagnostic imaging for submandibular duct atresia: literature review and case report. J Oral Maxillofac Surg. 70(12), 2819-22.

Neto, A. E. (2002). Sialolito na região de uma glândula parótida - relato de um caso clínico. BCI, 9 (35): $210-4$.

Neville, B. W., Damm, D. D., Allen, C. M., \& Bouquot, J. E. (2009). Patologia Oral e Maxilofacial, (3 ${ }^{\text {a }}$ ed.). Rio de Janeiro: Elsevier.

Pereira, A.S., Shitsuka, D. M., Parreira, F. J. \& Shitsuka, R. (2018). Metodologia da pesquisa científica. [e-book].Santa Maria. Ed.UAB/NTE/UFSM. https://repositorio.ufsm.br/bitstream/handle/1/15824/Lic Computacao_Metodologia-Pesquisa-Cientifica.pdf?sequence=1.

Pereira, R. V. S., Silva, J. de Ângelis A., Souza, J. R. dos S., Honorato, T. A. M., Silva, R. F. da, Andrade, C. E. de S., Campos, G. J. de L., \& Lucas Neto, A. (2020). Surgical treatment of submandibular salivary gland sialolith: case report. Research, Society and Development, $9(9)$, e829998072.

Rodrigues, G. H. C., Carvalho, V. J. G., Alves, F. A., \& Domaneschi, C. (2017). Giant submandibular sialolith conservatively treated. Autopsy Case Rep, $7(1): 9-11$

Siddiqui, S. J. (2002). Sialolithiasis: an unusually large submandibular salivary stone. Br Dent J, 193(2),89-91.

Silva, C. B., Cabral, L. N., Pinheiro, T. N., \& Vasconcellos II, A. J. A. (2021). Sialolitíase em região sublingual direita: relato de caso. Arch Health Invest, $10(3), 368-372$.

Silveira, R. L., Machado, R. A., Borges, H. O. I., \& Oliveira, R. B. (2005). Múltiplos sialolitos em glândula submandibular direita: relato de caso. Revista da Faculdade de Odontologia de Lins, 17(1), 39-42.

Souza, D. M. B., Silva, J. S. da, Nogueira, R. V. B., Vasconcellos, R. J. de H., \& Brêda Júnior, M. A. (2021). Caso atípico de múltiplos sialólitos no ducto da glândula submandibular. archives of health investigation, 10(6), 913-6.

Starling, C. R., Silva, D. T., \& Falcão A. F. P. (2012). Sialolitíase em glândula sublingual: relato de caso clínico. $R$ Ci med biol, 11(3), 346-50.

Torres, L. H. S., Santos, M. S., Diniz, J. A., Uchôa, C. P., Silva, J. A. A., Pereira Filho, V. A., \& Oliveira, E. D. (2019). Arch Health Invest, 8(8), 421-4. 